\title{
Electrical charging of helium and hydrogen droplets
}

\author{
Tito E. Huber and Frank Boccuzi \\ Polytechnic University, Brooklyn, New York, 11201 \\ E-mail: thuber@photon.poly.edu
}

The cryogenic fluids helium and hydrogen are versatile prototype fluids as they have important fundamental properties. Many of these properties are influenced by the boundary conditions that prevail at the container walls. These effects can be minimized in droplets. By creating a superfluid helium sample in the near-zero gravity of the orbiting shuttle, superfluid droplet dynamics can be studied for the first time. Also, the extent of supercooling of liquid hydrogen droplets can be investigated under such favorable conditions and we can attempt to make a superfluid molecular hydrogen droplet.

Changed droplets can be levitated using the quadrupole trap. In this paper we show that weakness of the electron bond to hydrogen and helium means that for a reasonable trapping field, the largest molecular hydrogen droplet that can be levitated on the ground is 6 micrometers in diameter. For helium, the maximum particle size is even smaller. Positive ions can strongly attach to helium and positively charged droplets appears possible on the ground. However, the «snowballs» produced by the ionic electrostriction are likely to pin and affect the superfluid from of helium. In particular, ions can compromise the proposed rotational superfluid dynamics experiments by nucleating vortices. Ions are also likely to spoil the supercooling of hydrogen. On the other hand, the droplets can be very large, macroscopic, under microgravity conditions. In fact, in that case the droplet diameter can be as large as $1 \mathrm{~mm}$ for hydrogen.

Specific issues discussed in this paper are, 1) injection and positioning of droplets if a microgravity environment and, 2) electronic and ionic charging and, 3) conditions of size and temperature for charge holding.

Although we specifically target the quadrupole trap, and the magnetic trap only indirectly, we will discuss that these work is relevant to all cryogenic droplet experiments in microgravity because the shuttle is a medium-to-high radiation environment and spontaneous charging of dielectric interfaces in an issue. 